\title{
Synovectomy during total knee arthroplasty: a pilot single-centre randomised controlled trial
}

\author{
Kenneth S. Rankin ${ }^{1 *}$ D, Jayasree Ramaskandhan ${ }^{1}$, Michelle Bardgett ${ }^{1}$, Katie Merrie ${ }^{1}$, Rajkumar Gangadharan ${ }^{1}$, \\ Ian Wilson ${ }^{2}$ and David Deehan ${ }^{1}$
}

\begin{abstract}
Background: Total knee arthroplasty (TKA) is an effective procedure for late-stage osteoarthritis (OA) of the knee; however, up to $20 \%$ of patients remain dissatisfied. In some patients, this may be due to residual inflammation of the synovium. Our aim was to perform the first randomised controlled trial (RCT) of synovectomy during TKA for patients with macroscopically inflamed synovium. The main objectives were to assess recruitment rates, protocol adherence and outcomes relating to safety such as haemoglobin decrease and adverse events. We also collected data on patient-reported outcomes.
\end{abstract}

Methods: We performed a single-centre pilot RCT. Patients with a macroscopically inflamed synovium were randomised to receive synovectomy versus a control group that did not undergo synovectomy. We determined feasibility by measuring patient enrolment, completeness of follow-up, and safety via haemoglobin decrease and documentation of adverse events.

Results: We screened 360 patients with 260 deemed ineligible or could not be recruited. From the 100 eligible patients, 54 were enrolled and 40 progressed through to randomisation. All made it to the 12-month follow-up, indicating good protocol adherence. There were no major differences in adverse events or haemoglobin decrease demonstrating acceptable safety. Outcomes relating to satisfaction were reliably obtained.

Conclusions: Patients with macroscopically inflamed synovium of the knee who are due to undergo TKA can be reliably recruited to a randomised trial and synovectomy can be performed safely. A large number is needed to be screened to identify eligible participants, and therefore, a multi-centre trial would be required to assess whether routine synovectomy would improve outcomes in these patients.

Trial registration: ISRCTN, ISRCTN31010214. Registered 6 October 2016-retrospectively registered

Keywords: Knee, Osteoarthritis, Synovectomy

\section{Background}

Osteoarthritis (OA) is the most common disease affecting synovial joints in the middle- to old-age population [1]. The knee is one of the most frequent anatomical locations involved, which results in a massive health economic burden related to physical disability [2]. Late-stage osteoarthritis of the knee is defined as severe pain not controlled with regular analgesic medication

\footnotetext{
* Correspondence: k.s.rankin@ncl.ac.uk

${ }^{1}$ Musculoskeletal Department, Freeman Hospital, High Heaton, Newcastle upon Tyne NE7 7DN, UK

Full list of author information is available at the end of the article
}

accompanied by radiographic evidence of joint space narrowing, osteophyte formation and subchondral sclerosis. Due to a complete lack of disease-modifying osteoarthritis agents, surgery in the form of total knee arthroplasty (TKA) remains the most effective method of managing late-stage cases [3]. There are an increasing number of patients receiving TKA which is due to improving knee implant designs and a lowering of thresholds for surgeons to offer surgery [4]. In the UK, over 85,000 patients undergo TKA each year [5], and in the USA, this figure is over 620,000 [6]. With the economic impact of the projected 56,918 patients requiring 
revision surgery which is due to reach $\$ 2$ billion annually by 2030, any reduction in the requirement for further procedures would be welcome [7].

Survival for most implant designs with revision taken as an endpoint is over 95\% at 10 years; however, satisfaction analyses indicate consistently that at least $20 \%$ of patients are dissatisfied with their knee replacement [8]. The variability in macroscopic appearance of the synovium has been noted by surgeons performing TKA [9], and the historical description of $\mathrm{OA}$ as a non-inflammatory disease has been superseded by clinical [10], radiological [11-14], and pathological [15] evidence of inflammatory processes, particularly in the knee joint. There is concern, therefore, that a proportion of patients reporting unsatisfactory outcomes following TKA may have persistent inflammatory activity in the knee joint driven by the presence of residual synovitis [16]. There have been three surgical trials assessing synovectomy during TKA. All concluded there is no benefit to performing synovectomy; however, in all of these studies, there was no intra-operative attempt to discriminate between inflamed and non-inflamed synovium [17-19].

Our aim was to perform the first randomised controlled trial (RCT) of synovectomy versus no synovectomy during TKA for OA in patients with macroscopically inflamed synovium. The main objectives were to assess recruitment rates, protocol adherence, and outcomes relating to safety such as haemoglobin decrease and adverse events. We also collected data on patient-reported outcomes.

\section{Methods}

\section{Study design and setting}

Ethical approval was obtained (Regional Ethics Committee reference: 10/H0904/76) followed by permission from our local Research Governance Department. The study set up commenced in June 2013, and final follow-up was in October 2015. Participants were recruited between 1 November, 2013, and 7 October, 2014. The study was performed at a university hospital in the UK. Forty patients who are due to undergo a routine primary total knee arthroplasty (TKA) procedure under the care of three senior surgeons completed participation in the study. The study is registered to the ISRCTN: 31010214. The schedule of the trial (Additional file 1) is available as supplementary material.

\section{Participants/study subjects}

Inclusion criteria were patients over the age of 18 years with a diagnosis of late-stage knee osteoarthritis. Exclusion criteria were as follows: refusal or inability to provide informed consent; those unable to answer questionnaires for cognitive reasons; patients diagnosed with auto-immune inflammatory arthritis of the knee; morbidly obese individuals (with $\mathrm{BMI}>40$ ); patients with a neuromuscular disorder that would affect their ability to mobilise; patients undergoing bilateral synchronous knee replacement procedures; patients undergoing more complex surgery requiring an implant with increased constraint including posterior cruciate sacrificing designs. Written consent was obtained from those agreeing to participate.

\section{Randomisation and blinding}

The trial consisted of two arms, and randomisation was performed intra-operatively by a research nurse attending the operating room. After eversion of the patella, the synovium was evaluated by the surgeon. Evidence of hyperplasia, papilla formation and engorgement was defined as being consistent with a macroscopically inflamed synovium and deemed an inclusion criterion (Fig. 1). Patients who did not have a macroscopically inflamed synovium underwent a standard surgical

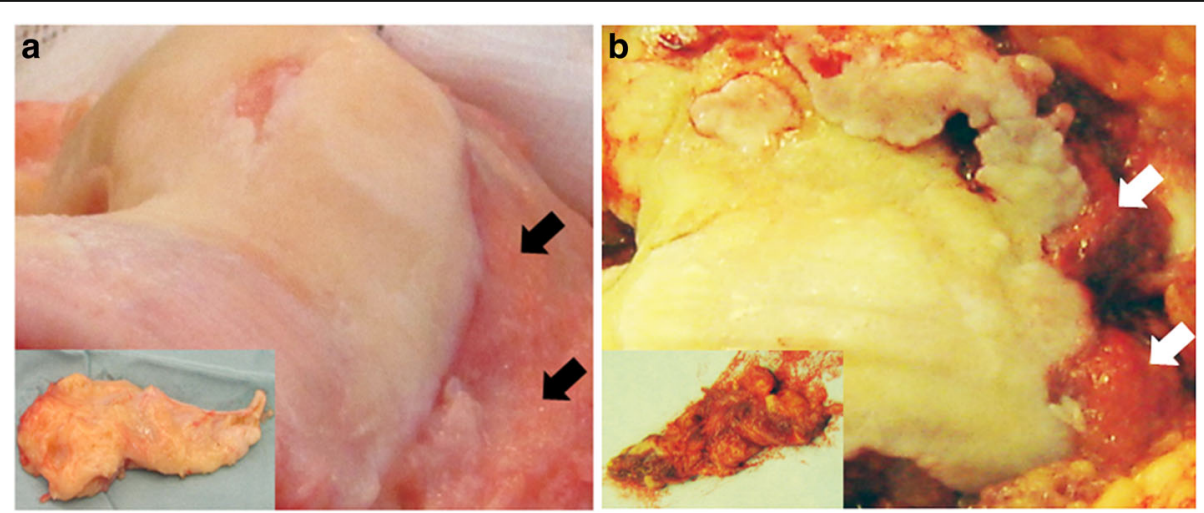

Fig. 1 Representative photographs of non-inflamed versus inflamed synovium. a Synovium that is not macroscopically inflamed. Black arrows: on the knee. Inset: small representative specimen. Patient is withdrawn from the study. b Synovium that is macroscopically inflamed. White arrows: on the knee. Inset: small representative specimen. Patient is randomised intra-operatively 
Table 1 Attrition from screening to enrolment

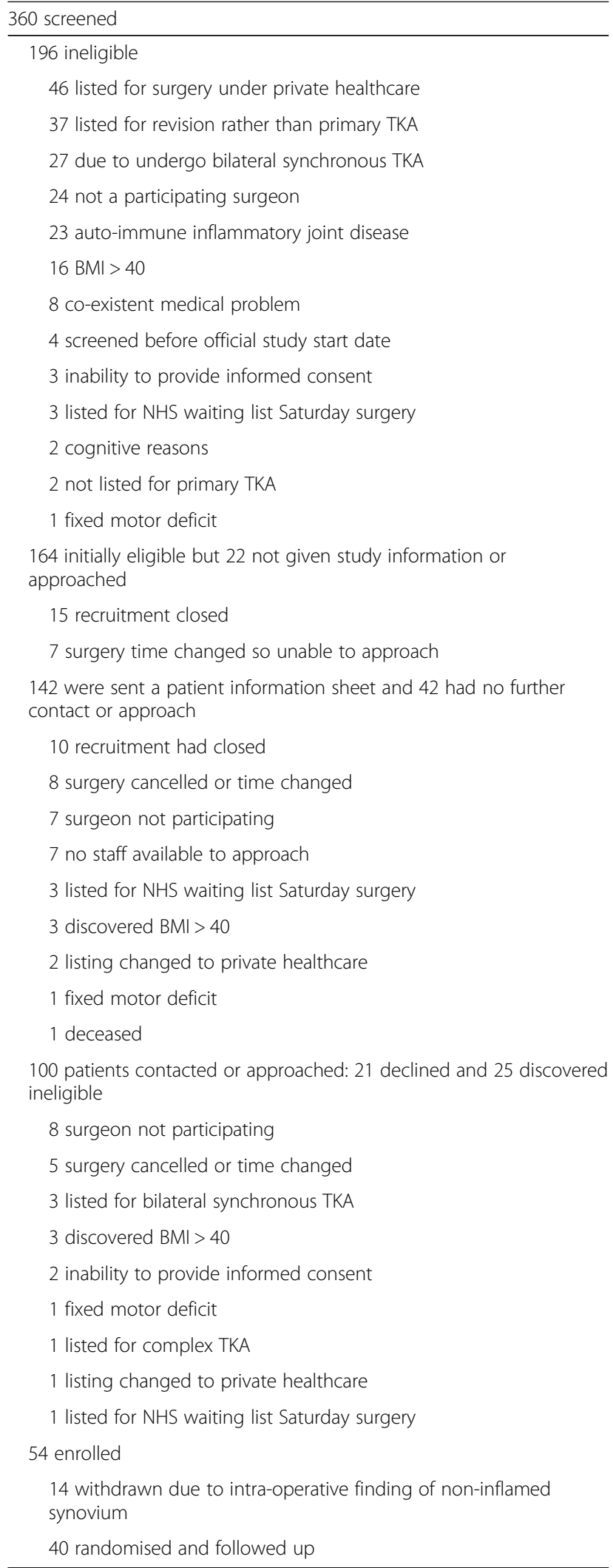

procedure and were withdrawn from the study. Participants with confirmed macroscopically inflamed synovium were randomised to receive synovectomy or no synovectomy and underwent a standard implantation of the prosthesis. Participants were blinded until their 1-year follow-up. Block randomisation was performed by a research nurse using the 'sealed envelope.com' website to provide the allocation until 20 participants had been recruited into each arm. The research nurse was not involved in any further aspects of the trial.

\section{Interventions}

All operations were performed using a standard surgical approach with prior application of a high thigh tourniquet. The procedure consisted of a midline incision, a medial parapatellar approach and eversion of the patella. Hoffa's fat pad in all cases was excised to facilitate access to the joint. A cemented cruciate retaining implant was used in all cases. No patients received tranexamic acid. Antibiotic and thromboprophylaxis protocols were identical for both groups, and post-operative rehabilitation was the same. For participants randomised to synovectomy, a meticulous technique was undertaken to avoid significant blood loss. This involved resection of the intimal and subintimal layers, leaving the vascular layer intact.

\section{Follow-up}

Our team of research physiotherapists followed participants at 6 weeks and 1 year to document clinical outcomes and patient-reported outcome measures. In addition, haemoglobin decrease day 1 post-operatively was documented. The clinical outcome measure was improvement in range of movement assessed pre-operatively and at 6 weeks and 1 year post-operatively. The patient-reported outcome measures evaluated included disease orientated, satisfaction and general quality of life scores. The Western Ontario and McMaster Universities osteoarthritis index (WOMAC) provides general information about the impact of the disease process on daily life [20] and was measured pre-operatively and 1 year post-operatively. A score for patient-reported co-morbid medical conditions was included to demonstrate that there were no participants with a disease process other than OA that would hinder their rehabilitation [21]. The Short Form 36 health survey (SF-36) [22], and EQ-5D [23] are general quality of life scores which have been demonstrated to improve following successful TKA and were therefore measured pre-operatively and 1 year post-operatively. Finally, a patient satisfaction from surgery score was obtained using a validated outcome measure [24]; this questionnaire includes four questions about satisfaction with overall outcome, pain relief, ability to perform activities of daily living and ability to participate in leisure 


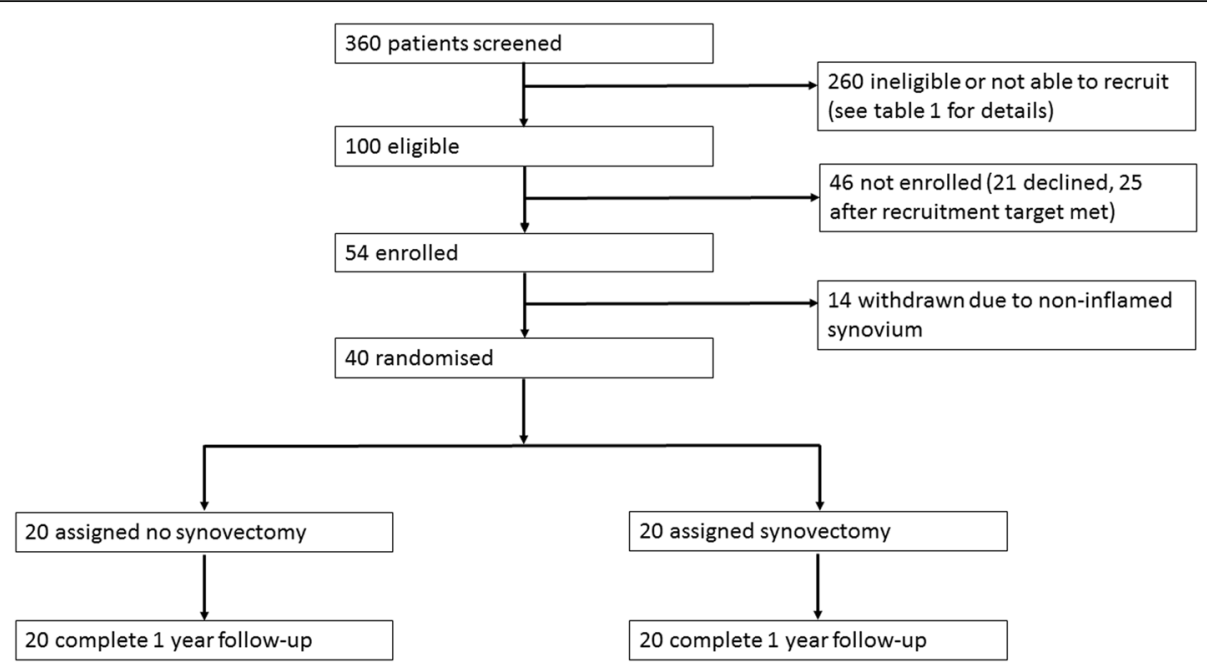

Fig. 2 CONSORT flow chart

activities. Responses are on a 4-point Likert scale, which ranges from very satisfied to very dissatisfied. The satisfaction score was measured at 1 year post-operatively. Complications were recorded using a standard proforma. Participants were given a telephone helpline number to contact the study team in the event of any adverse events. In addition, at the 6-week follow-up, the research team actively sought out any possible adverse events from the participants.

\section{Statistical analyses}

A sample size was not calculated due to the design of the study, i.e. pilot trial. The aim was to screen, enrol and randomise as many patients as possible over a 12-month recruitment period and stop recruitment when 20 to 30 patients were enrolled into each arm. Statistical analysis was performed on the clinical range

Table 2 Baseline characteristics of the participants according to intervention

No synovectomy $\left(n=\begin{array}{l}\text { Synovectomy }(n= \\ 20)\end{array}\right.$
$\begin{aligned} & \text { 20) }\end{aligned}$

\begin{tabular}{lll}
\hline & \multicolumn{2}{c}{ Sex } \\
Male, $n(\%)$ & $13(65 \%)$ & $16(80 \%)$ \\
Female, $n(\%)$ & $7(35 \%)$ & $4(20 \%)$ \\
Age in years (range) & $67.5(44.6-83.3)$ & $69.1(54.6-85.4)$ \\
$\begin{array}{l}\text { Average number of } \\
\text { Co-morbidities mean (SD) }\end{array}$ & $1.4(1.1)$ & $1.5(1.4)$ \\
$\begin{array}{l}\text { Side of operation } \\
\text { Right, } n(\%)\end{array}$ & $12(60 \%)$ & $7(35 \%)$ \\
Left, $n(\%)$ & $8(40 \%)$ & $13(65 \%)$ \\
BMI $\left(\mathrm{kg} / \mathrm{m}^{2}\right)$ mean & $28.7(3.2)$ & $30.1(3.4)$ \\
(SD) & & \\
\hline
\end{tabular}

of movement data to assess for improvement from the 6-week to 1-year time points which should be demonstrable in a small TKA group and also to assess for a difference between the intervention and no intervention groups. An analysis was also performed to check for a significant difference in haemoglobin decrease between the intervention and no intervention groups. The analysis was performed using SPSS software version 17 (SPSS Inc., Chicago, IL). The data were checked for normal distribution using the Kolmogorov-Smirnov test. The two sample $t$ test was used for comparison of means for the SF-36, WOMAC and EQ-5D scores. Results are presented with 95\% confidence intervals, and any hypothesis testing is preliminary and should be interpreted with caution.

\section{Results}

\section{Recruitment}

The participants were recruited between 1 November, 2013, and 7 October, 2014. Three hundred sixty patients were screened. Two hundred sixty were found to be ineligible or could not be recruited. Fifty-four of the eligible patients agreed to participate, and informed consent was obtained. A detailed summary of the attrition from screening is shown in Table 1.

Fourteen participants were subsequently withdrawn due to the finding of a non-inflamed synovium intra-operatively. Twenty participants were randomised to each group. Of the 40 participants, all were successfully followed up to the 1-year conclusion with no drop outs, as per the CONSORT diagram (Fig. 2).

\section{Baseline characteristics}

There were 40 patients in the study with 20 participants in each arm (Table 2). There were 29 males and 11 
females. Mean age was 67.5 for the no synovectomy group and 69.1 for the synovectomy group.

\section{Safety}

There was a decrease in mean haemoglobin levels observed in both groups from pre-operatively to day one post-operatively: no synovectomy $138 \mathrm{~g} / \mathrm{L}$ (SD 14) to $118 \mathrm{~g} / \mathrm{L}$ (SD 16) versus synovectomy $141 \mathrm{~g} / \mathrm{L}$ (SD 11) to $117 \mathrm{~g} / \mathrm{L}$ (SD 10). There was no difference in mean haemoglobin levels between the two groups both pre-operatively relative risk $-2.90,95 \% \mathrm{CI}-10.9$ to 5.1 , $p=0.467$, and post-operatively relative risk $0.30,95 \% \mathrm{CI}$ -8.36 to $8.96, p=0.944$. No patient received a blood transfusion.

There were 10 adverse events reported during the study (Table 3). Two were serious adverse events due to hospitalisation of the participants, both of whom were in the synovectomy group. In one case, this was due to constipation, and in the second case, the participant had developed a superficial wound infection. This was treated with antibiotics only and did not require surgical intervention. These serious adverse events were not classified as related to the synovectomy procedure.

\section{Feasibility}

All 40 randomised patients completed follow-up to the 1 -year end point. There was $100 \%$ documentation of range of movement scores at all time points.

For the patient-reported outcome measures, completeness of follow-up was $100 \%$ for the EQ-5D, $95 \%$ for the

Table 3 All adverse events

\begin{tabular}{lll}
\hline & $\begin{array}{l}\text { No synovectomy } \\
(n=20)\end{array}$ & $\begin{array}{l}\text { Synovectomy } \\
(n=20)\end{array}$ \\
\hline $\begin{array}{l}\text { Total adverse events } \\
\text { Serious adverse events }\end{array}$ & 3 & 7 \\
Death & 0 & 0 \\
Life threatening & 0 & 0 \\
Admission to hospital & 0 & 1 \\
Constipation & & Resolved with laxatives \\
Admission to hospital & 0 & 1 \\
$\begin{array}{l}\text { Superficial wound } \\
\text { infection }\end{array}$ & & Resolved with \\
Adverse events & & antibiotics \\
$\begin{array}{l}\text { Suspected DVT } \\
\text { (excluded) }\end{array}$ & 1 & 2 \\
$\begin{array}{l}\text { Suspected DVT } \\
\text { (confirmed) }\end{array}$ & 0 & 0 \\
Hip pain & & 1 \\
Nausea & 1 & 0 \\
Wound problem & 0 & 1 \\
Urinary tract infection & 0 & 1 \\
\hline
\end{tabular}

WOMAC and SF-36 scores and $87.5 \%$ for the patient satisfaction from surgery scores (Table 4). The loss of data was due to illegible recording on the scoresheet by the participants. There were no protocol deviations.

\section{Secondary outcomes}

There was no difference in range of movement within the groups from pre-operatively to 1 year post-operatively $(p=0.602)$. There was a significant improvement in knee range of motion from 6 weeks post-operatively to 1 year post-operatively for both groups (no synovectomy: $100.5^{\circ}$ (SD 14.1) to $113.8^{\circ}$ (SD 12.6), $p<0.0001$; synovectomy: $97.5^{\circ}$ (SD 12.5) to $114.2^{\circ}$ (SD 11.4), $p<0.0001)$. For the EQ-5D Health score, the no synovectomy group scored lower than the synovectomy group at 1 year ( 81.00 versus 85.63$)$, but this did not achieve statistical significance (Table 5).

There was a significant improvement in mean SF-36 scores from pre-operative to 1 year post-operatively for all domains $(p<0.0001)$ within both groups. There was no statistically significant difference between the groups at the 1-year follow-up (Table 6).

There was a significant improvement in mean WOMAC scores for the no synovectomy group (pain 39.7 (SD 15.3) to 78.6 (SD 21.0); function 40.3 (SD 19.3) to 74.3 (SD 23.3); stiffness 40.1 (SD 21.4) to 76.3 (SD $19.5) ; p<0.0001$ ) and the synovectomy group (pain 43.6 (SD 15.0) to 83.9 (SD 21.2); function 48.1 (SD 11.6) to 84.6 (SD 16.6); stiffness 50.0 (SD 18.1) to 80.1 (SD 15.3); $p<0.0001)$. There was no significant difference between groups in mean WOMAC scores for pain $(p=0.448)$, function $(p=0.131)$ and stiffness $(p=0.531)$ at 1 year post-operatively (Table 7).

There was no difference between groups in terms of patient satisfaction reported for pain relief (synovectomy group $94.1 \%$ vs control group $88.9 \%$; proportion difference $=5.2 \%$, 95\% CI $63 \%$ to $98 \%$ ), return to activities of daily living (ADL) (synovectomy group $83.4 \%$ vs control group 88.5\%; proportion difference $=5.1 \%$, 95\% CI $60 \%$ to $90 \%$ ), return to recreational activities (synovectomy group $77.8 \%$ vs control group $100 \%$; proportion difference $=22.2 \%$, 95\% CI $54 \%$ to $91 \%)$ and overall satisfaction from surgery (synovectomy group $89.9 \%$ vs control

Table 4 Completeness of follow-up

\begin{tabular}{llll}
\hline Outcome & Pre-operative, $n(\%)$ & 6 weeks, $n(\%)$ & 1 year, $n(\%)$ \\
\hline Range of movement & $40(100)$ & $40(100)$ & $40(100)$ \\
WOMAC & $38(95)$ & $\mathrm{n} / \mathrm{a}$ & $38(95)$ \\
SF-36 & $38(95)$ & $\mathrm{n} / \mathrm{a}$ & $38(95)$ \\
EQ-5D & $40(100)$ & $\mathrm{n} / \mathrm{a}$ & $40(100)$ \\
Satisfaction score & $\mathrm{n} / \mathrm{a}$ & $\mathrm{n} / \mathrm{a}$ & $35(87.5)$
\end{tabular}

WOMAC Western Ontario and McMaster Universities Arthritis Index, SF-36 Short form 36, EQ-5D Euroqol Five Dimension Scale 
Table 5 EQ-5D pre-operatively and 1 year post-operatively. Numbers are mean (SD)

\begin{tabular}{llll}
\hline & No synovectomy & Synovectomy & $95 \% \mathrm{Cl}$ \\
\hline Pre-operative & $n=20$ & $n=20$ & $0.50(-10.29$ to 11.29) \\
& $77.55(17.57)$ & $77.05(16.07)$ & \\
One year & $n=19$ & $n=16$ & -4.63 (- 14.07 to 4.82) \\
post-operative & $81.00(14.15)$ & $85.63(13.26)$ & \\
\hline
\end{tabular}

$E Q-5 D$ Euroqol five dimension scale, $S D$ standard deviation, $C l$ confidence interval

group 93.8\%; proportion difference $=3.9 \%$, 95\% CI $67 \%$ to $96 \%$ ) at 1 year post-operatively (Figs. 3 and 4 ).

\section{Discussion}

The evidence of an inflammatory component of knee $\mathrm{OA}$ at the early to late stages of disease is increasing and can even be tracked systemically [25]. At the knee joint, apart from the synovium, there are studies indicating that other tissues such as the fat pad can be a source of inflammation [26]. Furthermore, genomic studies of various joint tissue types from patients with knee OA have implicated epigenetic effects that result in the upregulation of pro-inflammatory cytokines [27] and the link from inflammation to the stimulation of pain pathways in knee OA is well established [28]. From a clinical perspective, a case series of arthroscopic debridement and synovectomy in the moderate to severe stages of knee OA has shown some benefit; however, this was temporary due to the progressive nature of the pathology [29]. Therefore, TKA remains the treatment of choice for late-stage disease but the significant proportion of dissatisfied patients continues to be a challenge. The aim of future therapy for knee OA should include accurate identification of those patients who are at risk of residual pain due to persistent inflammation in the soft tissues following TKA. This would

Table 6 SF-36 1 year post-operatively. Numbers are mean (SD)

\begin{tabular}{llll}
\hline SF-36 domains & No synovectomy & Synovectomy & $95 \% \mathrm{Cl}$ \\
\hline Physical & $n=19$ & $n=18$ & -14.10 \\
functioning & $60.0(26.2)$ & $74.1(25.2)$ & $(-31.29$ to 3.08) \\
Role-physical & $n=19$ & $n=19$ & -13.20 \\
& $64.5(33.1)$ & $77.6(29.0)$ & $(-33.60$ to 7.30$)$ \\
Bodily pain & $n=19$ & $n=19$ & -13.11 \\
& $59.2(30.8)$ & $72.3(25.1)$ & $(-31.62$ to 5.41$)$ \\
General & $n=19$ & $n=19$ & 4.55 \\
health & $66.3(21.7)$ & $61.7(23.1)$ & $(-10.22$ to 19.31$)$ \\
Vitality & $n=19$ & $n=19$ & -4.28 \\
& $60.2(18.3)$ & $64.5(23.4)$ & $(-18.12$ to 9.57$)$ \\
Social & $n=19$ & $n=19$ & -13.82 \\
functioning & $71.1(24.7)$ & $84.9(23.0)$ & $(-29.54$ to 1.91$)$ \\
Role-emotional & $n=19$ & $n=19$ & 4.82 \\
& $86.8(25.8)$ & $82.0(30.1)$ & $(-13.63$ to 23.28) \\
Mental health & $n=19$ & $n=19$ & -2.96 \\
& $77.9(18.5)$ & $80.9(17.5)$ & $(-14.82$ to 8.90$)$ \\
\hline
\end{tabular}

SF-36 Short Form 36, SD standard deviation, $\mathrm{Cl}$ confidence interval
Table 7 WOMAC scores 1 year post-operatively. Numbers are mean (SD)

\begin{tabular}{llll}
\hline & No synovectomy & Synovectomy & $95 \% \mathrm{Cl}$ \\
\hline Pain & $n=19$ & $n=19$ & $-5.26(-19.20$ to 8.67$)$ \\
& $78.6(21.0)$ & $83.9(21.2)$ & \\
Function & $n=19$ & $n=18$ & $-10.29(-28.30$ to 3.22$)$ \\
& $74.3(19.3)$ & $84.6(16.6)$ & \\
Stiffness & $n=18$ & $n=17$ & $-3.76(-15.86$ to 8.34$)$ \\
& $76.3(19.5)$ & $80.1(15.3)$ & \\
\hline
\end{tabular}

WOMAC Western Ontario and McMaster Universities Arthritis Index, SD

standard deviation, $\mathrm{Cl}$ confidence interval

involve assessing clinical, radiological and possibly systemic parameters to define the level of inflammation in the joint prior to embarking on therapy. There have been three randomised controlled trials that have sought to assess the effect of synovectomy during TKA [17-19]. In one of these studies, patients with clinical evidence of synovial thickening were included, but there was no mention of the state of the synovium intra-operatively [18]. The other two studies made no attempt to assess levels of inflammation in the joint $[17,19]$. These trials did not discern any benefit from synovectomy, and in two studies, there was evidence of increased blood loss in the intervention group [17, 19]. In one of these trials [17], the participants received bilateral synchronous knee replacements with one side randomised to undergo synovectomy. Blood loss was $107 \mathrm{cc}$ higher in the synovectomy patients, and this did not impact on outcomes. It should be noted that the surgical technique for synovectomy was described in only one of these trials which entailed resection of all the synovial layers, including the vasculature [18]. This is important, because an accurate synovectomy technique that removes the subintimal and intimal layers but leaves the vascular layer intact will result in minimal haemorrhage if the precise tissue planes are identified and respected. In our study, we randomised the participants intra-operatively after confirmation of the macroscopically inflamed synovium and performed a meticulous synovectomy technique retaining the vascular layer. Our trial demonstrates that synovectomy can be performed safely in patients with evidence of synovitis with no detrimental effects. We have also demonstrated that the enrolled participants could be followed up reliably up to 1 year post-operatively with documentation of adverse events and patient-reported outcome measures. In keeping with the difficulties experienced in recruiting to surgical RCTs in general, our study reveals a high rate of attrition from screening to enrolment with a wide range of reasons for eligible patients not making it to the consent stage. This is 


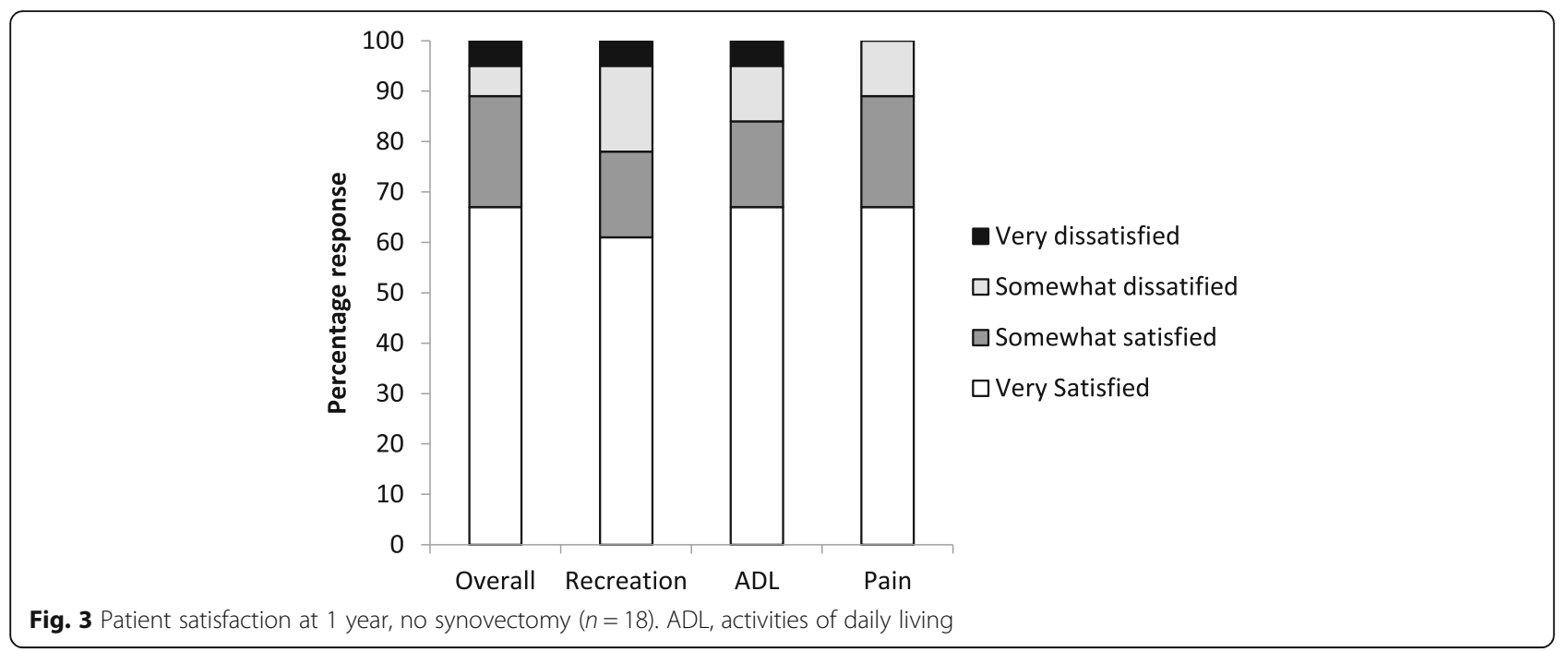

important because a subsequent trial would need to recruit from multiple centres in order to ensure that the required number of participants to address the potential superiority of the synovectomy intervention would be achieved in a reasonable timeframe. Options for reducing attrition could also be considered such as ensuring that provision is made in subsequent funding applications for the definitive trial for additional staff to screen more effectively and prevent drop out from identification of eligibility to participant approach and enrolment. A further issue was some inconsistency at follow-up. This was mainly due to illegible scores from the patient questionnaires. This could be mitigated by ensuring additional resources are available to request a further score from a participant when the initial submission is not evaluable. A weakness of our study is that the assessment of the synovium was based on a colour judgement by the surgeon with no imaging or tissue sampling to correlate, and therefore, further studies are already underway at our institution to assess whether the synovial tissue judged to be macroscopically inflamed correlates with pre-operative imaging and tissue profiles. A further limitation of our trial is the small sample size which means that all the results should be interpreted with caution. Regarding synovectomy during knee replacement, large multi-centre surgical studies are warranted to assess the potential benefits in larger patient cohorts. Future trial design should include objective measures of inflammation both in the joint tissues and systemically. For those patients that still suffer from pain due to residual inflammation following TKA, adjunctive therapy in the form of biologics that affect relevant cellular pathways is an option to be explored as evidenced by the use of anakinra to

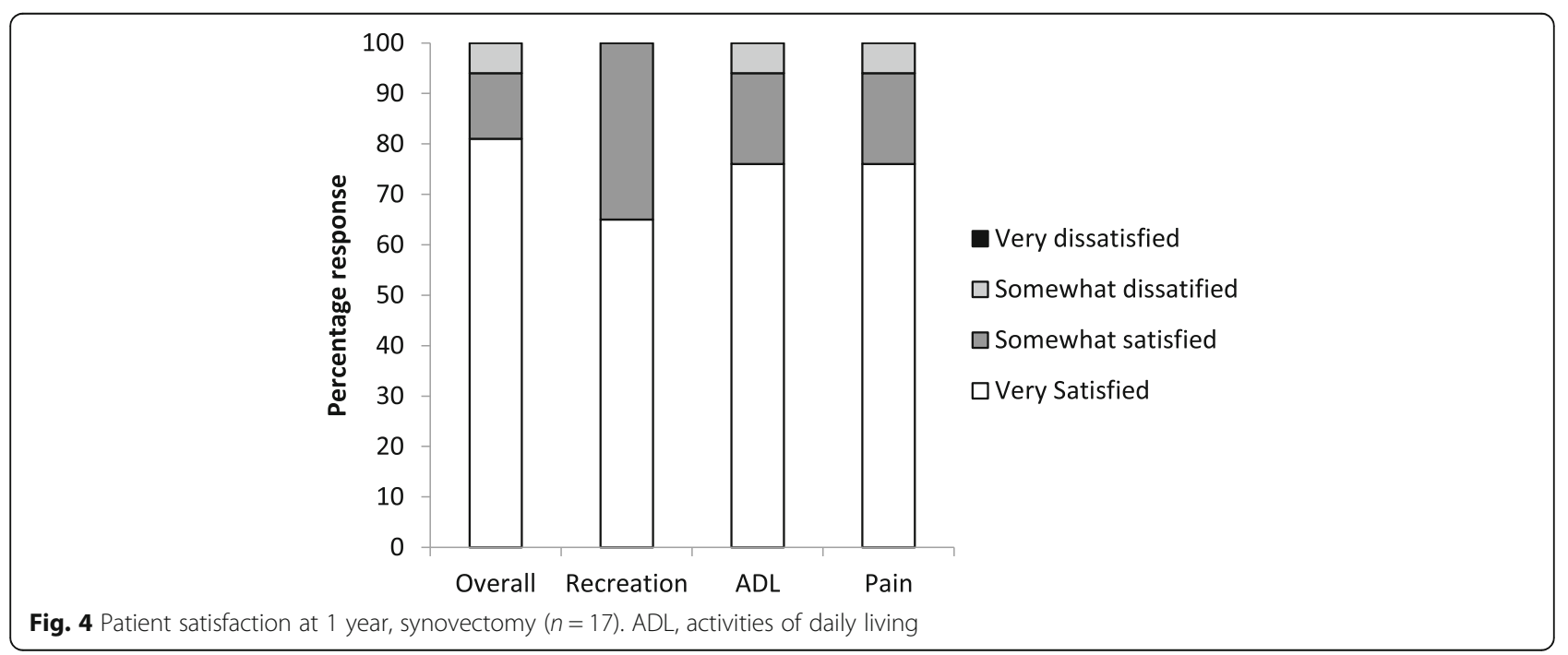


improve symptomatology in patients with arthrofibrosis following TKA [30]. For patients with severe pain likely related to synovitis but with minimal changes on the plain radiograph, a combined approach using arthroscopic synovectomy and medical therapies may avoid the requirement for joint replacement. Defining these treatment strategies will require close collaboration between orthopaedic surgeons and rheumatologists in order to design studies which will allow us to develop personalised multi-modal therapeutic for this large group of patients with an awareness that recruiting to these studies will require significant amounts of funding with multi-centre involvement.

\section{Additional file}

Additional file 1: Schedule of the trial. (PPT $259 \mathrm{~kb}$ )

\section{Abbreviations}

RCT: Randomised controlled trial; TKA: Total knee arthroplasty

\section{Acknowledgements}

We would like to acknowledge the contribution of the research nurses and physiotherapists at the Freeman Hospital, Newcastle, upon Tyne who contributed to screening patients.

\section{Funding}

This work was supported by the Research Capability Funding from the United Kingdom National Institute for Health Research.

\section{Availability of data and materials}

The datasets during and/or analysed during the study are available from the corresponding author on reasonable request.

\section{Authors' contributions}

$J R$ and $M B$ recruited the participants and performed the follow-up. KR, RG and DD performed the surgery. KM performed all the key data administration tasks. IW performed the statistical analyses. All authors contributed to the writing editing and formatting of the manuscript. All authors read and approved the final manuscript.

\section{Ethics approval and consent to participate}

Ethical approval was obtained (Regional Ethics Committee reference: 10/ H0904/76), and all participants provided informed consent.

\section{Consent for publication}

The images in Fig. 1 were taken after informed consent was obtained using the standard form at our institution.

\section{Competing interests}

The authors declare that they have no competing interests.

\section{Publisher's Note}

Springer Nature remains neutral with regard to jurisdictional claims in published maps and institutional affiliations.

\section{Author details}

${ }^{1}$ Musculoskeletal Department, Freeman Hospital, High Heaton, Newcastle upon Tyne NE7 7DN, UK. ${ }^{2}$ Institute of Genetic Medicine, University of Newcastle upon Tyne, International Centre for Life, Central Parkway, Newcastle upon Tyne NE1 3BZ, UK.
Received: 12 March 2018 Accepted: 15 August 2018

Published online: 25 August 2018

\section{References}

1. Glyn-Jones S, Palmer AJ, Agricola R, Price AJ, Vincent TL, Weinans $H$, Osteoarthritis CAJ. Lancet. 2015;386:376-87.

2. Agaliotis M, Mackey MG, Jan S, Fransen M. Burden of reduced work productivity among people with chronic knee pain: a systematic review. Occup Environ Med. 2014;71:651-9.

3. Fibel $\mathrm{KH}$, Hillstrom $\mathrm{HJ}$, Halpern BC. State-of-the-art management of knee osteoarthritis. World J Clin Cases. 2015;16:89-101.

4. Losina E, Thornhill TS, Rome BN, Wright J, Katz JN. The dramatic increase in total knee replacement utilization rates in the United States cannot be fully explained by growth in population size and the obesity epidemic. J Bone Joint Surg Am. 2012;94:201-7.

5. http://www.njrreports.org.uk/Portals/0/PDFdownloads/ NJR\%2014th\%20Annual\%20Report\%202017.pdf Last accessed 26 February, 2018.

6. Weinstein AM, Rome BN, Reichmann WM, Collins JE, Burbine SA, Thornhill TS, Wright J, Katz JN, Losina E. Estimating the burden of total knee replacement in the United States. J Bone Joint Surg Am. 2013;95:385-92.

7. Lavernia C, Lee DJ, Hernandez VH. The increasing financial burden of knee revision surgery in the United States. Clin Orthop Relat Res. 2006:446:221-6.

8. Maratt JD, Lee YY, Lyman S, Westrich GH. Predictors of satisfaction following Total knee Arthroplasty. J Arthroplast. 2015;30:1142-5.

9. Stoppiello LA, Mapp PI, Wilson D, Hill R, Scammell BE, Walsh DA. Structural associations of symptomatic knee osteoarthritis. Arthritis Rheumatol. 2014; 66:3018-27.

10. Ayral X, Pickering EH, Woodworth TG, Mackillop N, Dougados M. Synovitis: a potential predictive factor of structural progression of medial tibiofemoral knee osteoarthritis - results of a 1 year longitudinal arthroscopic study in 422 patients. Osteoarthr Cartil. 2005;13:361-7.

11. Atukorala I, Kwoh CK, Guermazi A, Roemer FW, Boudreau RM, Hannon MJ, Hunter DJ. Synovitis in knee osteoarthritis: a precursor of disease? Ann Rheum Dis. 2016:75:390-5.

12. Hill CL, Hunter DJ, Niu J, Clancy M, Guermazi A, Genant H, Gale D, Grainger A, Conaghan P, Felson DT. Synovitis detected on magnetic resonance imaging and its relation to pain and cartilage loss in knee osteoarthritis. Ann Rheum Dis. 2007:66:1599-603.

13. Roemer FW, Guermazi A, Felson DT, Niu J, Nevitt MC, Crema MD, Lynch JA, Lewis CE, Torner J, Zhang Y. Presence of MRI-detected joint effusion and synovitis increases the risk of cartilage loss in knees without osteoarthritis at 30-month follow-up: the MOST study. Ann Rheum Dis. 2011;70:1804-9.

14. Song $\mathbb{H}_{\text {, Althoff } C E}$, Hermann KG, Scheel AK, Knetsch T, Schoenharting M, Werner C, Burmester GR, Backhaus M. Knee osteoarthritis. Efficacy of a new method of contrast-enhanced musculoskeletal ultrasonography in detection of synovitis in patients with knee osteoarthritis in comparison with magnetic resonance imaging. Ann Rheum Dis. 2008;67:19-25.

15. Scanzello CR, Goldring SR. The role of synovitis in osteoarthritis pathogenesis. Bone. 2012;51:249-57.

16. Gonzalez MH, Mekhail AO. The failed total knee arthroplasty: evaluation and etiology. J Am Acad Orthop Surg. 2004;12:436-46.

17. Kilicarslan K, Yalcin N, Cicek H, Dogramaci Y, Ugurlu M, Ozkan H, Yildirim H. The effect of total synovectomy in total knee arthroplasty: a prospective randomized controlled study. Knee Surg Sports Traumatol Arthrosc. 2011;19: 932-5.

18. Tanavalee A, Honsawek S, Rojpornpradit T, Sakdinakiattikoon M, Ngarmukos S. Inflammation related to synovectomy during total knee replacement in patients with primary osteoarthritis: a prospective, randomised study. J Bone Joint Surg (Br). 2011:93:1065-70.

19. Zhaoning $X, X u Y$, Shaoqi T, Baiqiang $H$, Kang $S$. The effect of synovectomy on bleeding and clinical outcomes for total knee replacement. Bone Joint J. 2013:95-B:1197-200.

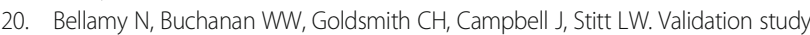
of WOMAC: a health status instrument for measuring clinically important patient relevant outcomes to antirheumatic drug therapy in patients with osteoarthritis of the hip or knee. J Rheumatol. 1988;15:1833-40.

21. Sangha O, Stucki G, Liang MH, Fossel AH, Katz JN. The Self-Administered Comorbidity Questionnaire: a new method to assess comorbidity for clinical and health services research. Arthritis Rheum. 2003;49:156-63. 
22. McHorney CA, Ware JE Jr, Lu JF, Sherbourne CD. The MOS 36-item ShortForm Health Survey (SF-36): III. Tests of data quality, scaling assumptions, and reliability across diverse patient groups. Med Care. 1994;32:40-66.

23. Giesinger JM, Hamilton DF, Jost B, Behrend H, Giesinger KWOMAC. EQ-5D and Knee Society Score thresholds for treatment success after total knee arthroplasty. J Arthroplast. 2015;30:2154-8.

24. Mahomed N, Gandhi R, Daltroy L, Katz JN. The self-administered patient satisfaction scale for primary hip and knee arthroplasty. Arthritis. 2011;2011: 591253 https://doi.org/10.1155/2011/591253.

25. Attur M, Statnikov A, Samuels J, Li Z, Alekseyenko AV, Greenberg JD, Krasnokutsky S, Rybak L, Lu QA, Todd J, Zhou H, Jordan JM, Kraus VB, Aliferis CF, Abramson SB. Plasma levels of interleukin-1 receptor antagonist (IL1Ra) predict radiographic progression of symptomatic knee osteoarthritis. Osteoarthr Cartil. 2015;23:1915-24.

26. Ioan-Facsinay A, Kloppenburg M. An emerging player in knee osteoarthritis: the infrapatellar fat pad. Arthritis Res Ther. 2013;15:225.

27. Rogers EL, Reynard LN, Loughlin J. The role of inflammation-related genes in osteoarthritis. Osteoarthr Cartil. 2015;23:1933-8.

28. Neogi T, Guermazi A, Roemer F, Nevitt M, Scholz J, Arendt-Nielsen L, Woolf C, Niu J, Bradley LA, Quinn E, Frey Law L. Association of joint inflammation with pain sensitization in knee osteoarthritis: the Multicenter Osteoarthritis Study. Arthritis Rheumatol. 2016;68:654-61.

29. Steadman JR, Ramappa AJ, Maxwell RB, Briggs KK. An arthroscopic treatment regimen for osteoarthritis of the knee. Arthroscopy. 2007;23:948-55.

30. Dixon D, Coates J, del Carpio Pons A, Horabin J, Walker A, Abdul N, Kalson NS, Brewster NT, Weir DJ, Deehan DJ, Mann DA, Borthwick LA. A potentia mode of action for anakinra in patients with arthrofibrosis following total knee arthroplasty. Sci Rep. 2015;10:16466.

Ready to submit your research? Choose BMC and benefit from:

- fast, convenient online submission

- thorough peer review by experienced researchers in your field

- rapid publication on acceptance

- support for research data, including large and complex data types

- gold Open Access which fosters wider collaboration and increased citations

- maximum visibility for your research: over $100 \mathrm{M}$ website views per year

At $\mathrm{BMC}$, research is always in progress.

Learn more biomedcentral.com/submissions 